\title{
The Compound Binomial Risk Model with Randomly Charging Premiums and Paying Dividends to Shareholders
}

\author{
Xiong Wang and Lei He \\ School of Business, Central South University, Changsha, Hunan 410083, China \\ Correspondence should be addressed to Xiong Wang; wx2011@csu.edu.cn
}

Received 24 October 2012; Revised 22 May 2013; Accepted 4 June 2013

Academic Editor: Samir H. Saker

Copyright (C) 2013 X. Wang and L. He. This is an open access article distributed under the Creative Commons Attribution License, which permits unrestricted use, distribution, and reproduction in any medium, provided the original work is properly cited.

\begin{abstract}
Based on characteristics of the nonlife joint-stock insurance company, this paper presents a compound binomial risk model that randomizes the premium income on unit time and sets the threshold $x$ for paying dividends to shareholders. In this model, the insurance company obtains the insurance policy in unit time with probability $p_{0}$ and pays dividends to shareholders with probability $p_{1}$ when the surplus is no less than $x$. We then derive the recursive formulas of the expected discounted penalty function and the asymptotic estimate for it. And we will derive the recursive formulas and asymptotic estimates for the ruin probability and the distribution function of the deficit at ruin. The numerical examples have been shown to illustrate the accuracy of the asymptotic estimations.
\end{abstract}

\section{Introduction}

The compound binomial risk model is one of the classical actuarial models that have been studied extensively. The classic literatures about the compound risk model primarily include [1-9]. With the emergence and development of dividend insurance, compound binomial risk models considering the case of paying dividends to policyholder are attracting more and more attention of actuarial scholars; see [10-14]. Reference [10] builds the compound binomial risk model with randomly dividends payment and derives the ruin problem by recursive algorithm for cases where the company pays dividends to its policyholders with a certain probability. Reference [11] obtains and solves two defective renewal equations for the Gerber-Shiu penalty function under the compound binomial model proposed in [10]. Reference [12] generalizes the model of [10] and derives the discounted penalty function under the compound binomial model with a multithreshold dividend structure and randomized dividend payments. Considering the fact that the joint-stock company may pay dividends to the policyholders and shareholders, [13] builds the compound binomial risk model with random dividends payment to the policyholders and shareholders and studies the ruin problem with the model. furthermore, [14] derives the arbitrary moments of discounted dividend payments under the compound binomial risk model with interest on the surplus and periodically paying dividend.

The previously mentioned models are compound binomial risk models with a constant premium rate suited for depicting the surplus of the life insurance companies which collect installment premiums. However, nonlife insurance companies (e.g., property insurance companies) charge premiums immediately, and insurance policies are obtained randomly in unit time. Thus the model with a constant premium rate cannot reasonably describe the surplus of the nonlife insurance companies. Moreover, joint-stock nonlife insurance companies need to pay dividends to the shareholders randomly (see [10]). However, these characteristics have not been considered in [10] together. Thus, [10] cannot be suitable for describing the surplus of the joint-stock nonlife insurance companies. In order to address the deficiencies of the models in [10], a compound binomial risk model has been developed with random premiums and dividends payment to shareholders, and it derives the recursive formulas and asymptotic estimates of the ruin probability and the distribution of the deficit at ruin.

This paper is organized as follows. In Section 2, we build the compound binomial risk model with randomly charging premiums and paying dividends to shareholders. In Section 3 , we derive the recursive formulas of discounted penalty 
function. In Section 4, we derive the asymptotic estimates for the discounted penalty function. In Section 5, we obtain recursive formulas, and asymptotic estimates of the ruin probability and the distribution function of deficit at ruin are obtained. Finally, the conclusion is shown in Section 6.

\section{The Model and Preliminaries}

Consider the compound binomial model with randomized decisions on paying dividends, which is described by

$$
U(t)=u+t-S(t)-D(t), \quad t=1,2, \ldots,
$$

with the initial surplus of the insurance $u(\geq 0) . S_{t}$ is the aggregate claim up to time $t$; that is,

$$
S(t)=\sum_{k=1}^{t} \theta_{k} X_{k}, \quad t=1,2, \ldots,
$$

where $\theta_{t}$ denotes whether the claim occurs or not in $(t-1, t]$; the event in which the claim occurs is denoted by $\theta_{t+1}=1$; the event in which no claim occurs is denoted by $\theta_{t+1}=0$. The probability of a claim is $p$ and the probability of no claim is $q=1-p$ in any period $(t, t+1] . \theta=\left\{\theta_{t}, t=1,2, \ldots\right\}$ is independent and identically distributed random series. $X=$ $\left\{X_{t}, t=1,2, \ldots\right\}$ is independent and identically distributed as $F=\{p(k)=\operatorname{Pr}(X=k) ; k=1,2, \ldots\} . D(t)$ is the aggregate dividends payment; that is,

$$
D(t)=\sum_{k=1}^{t} \epsilon_{k} I(U(k-1) \geq x), \quad t=1,2, \ldots,
$$

where $x(>0)$ is the threshold such that the insurance company may pay dividends to the shareholders, and $I(B)$ is the indicator function of a set $B . \epsilon_{t}$ denotes whether dividend is paid or not in $(t-1, t]$. When the surplus is no less than $x$, the company pays one dividend to the shareholders with probability $p_{1}$ (denoted by $\epsilon_{t}=1$ ) and not with the probability $q_{1}=1-p_{1}\left(\right.$ denoted by $\left.\epsilon_{t}=0\right) ; \epsilon=\left\{\epsilon_{t}, t=\right.$ $1,2, \ldots\}$ is independent and identically distributed as $B\left(p_{1}\right)$ $\left(0<p_{1}<1\right)$.

According to the feature of the variety of the surplus in joint-stock nonlife insurance companies, we further assume that premium is charged randomly in unit period $(t-1, t]$. Then the aggregate premium is

$$
M(t)=\sum_{k=1}^{t} I_{k}, \quad t=1,2, \ldots,
$$

where $I_{t}$ is variable with distribution $B\left(p_{0}\right)\left(0<p_{0} \leq 1\right)$, $q_{0}=1-p_{0}$, and denotes obtaining an insurance policy by $I_{t}=1$ in $(t-1, t]$, as well as denotes not obtaining an insurance policy by $I_{t}=0 . I=\left\{I_{t}, t=1,2, \ldots\right\}$ is independent and identically distributed. Furthermore, the random series $\theta, X, \epsilon, I$ are assumed to be mutually independent. Then, the surplus of the nonlife insurance joint-stock company is

$$
\begin{gathered}
U(0)=u \\
U(t)=u+M(t)-S(t)-D(t), \quad t=1,2, \ldots
\end{gathered}
$$

The model is called as the compound binomial risk model with random premiums and dividends payment to shareholders.

Remark. (1) Model (5) is the general form of the model in [10] and is the model in [10] if $p_{0}=1$. And also, model (5) can be regarded as general form of the classic risk model and exactly the classic risk model if $p_{0}=1, p_{1}=0$.

(2) In this model, the initial time $t=0$ is some time in the past at which point we begin studying the surplus of the jointstock nonlife insurance company, but not the time when the company is created.

Define ruin time with $T=\inf \{t \geq 0 \mid U(t)<0\}$. The ultimate ruin probability is defined by $\psi(u)=\operatorname{Pr}(T<+\infty$ | $U(0)=u)$. Define the (expected discounted) penalty function by

$$
\phi_{r}(u)=E\left[f\left(U_{T-1},\left|U_{T}\right|\right) I(T<+\infty) r^{-T} \mid U(0)=u\right] \text {, }
$$

where $f(x, y)(x \geq 0, y \geq 0)$ is the non-negative bounded function, $0<r \leq 1$. In this paper, the fact that $\sum_{k=0}^{-1} m_{k}=0$ is adopted.

Let $P(n)=\sum_{k=1}^{n} p(k), \bar{P}(n)=1-P(n)$. We always assume that $\mu=\sum_{k=1}^{+\infty} k p(k)=\sum_{k=0}^{+\infty} \bar{P}(n)<\infty$, and the $E\left[I_{k}-\theta_{k} X_{k}-\right.$ $\left.\epsilon_{k}\right]=p_{0}-p \mu-p_{1}>0$, which leads to the positive security loading. Denote that the security loading $\delta: \delta=\left(p_{0}-p \mu-\right.$ $\left.p_{1}\right) / p \mu>0$. Let $\phi(u)=\phi_{1}(u)$.

\section{The Recursive Formulas of the Penalty Function}

Theorem 1. Let $J(x)=q_{0} p_{1} p(x)+\left(q_{0} q_{1}+p_{0} p_{1}\right) p(x+1)+$ $p_{0} q_{1} p(x+2), \bar{J}(x)=q_{0} p_{1} \bar{P}(x)+\left(q_{0} q_{1}+p_{0} p_{1}\right) \bar{P}(x+1)+$ $p_{0} q_{1} \bar{P}(x=2), T(x)=p_{0} p(x+1)+q_{0} p(x), \bar{T}(x)=p_{0} \bar{P}(x+$ 1) $+q_{0} \bar{P}(x)$. Then

(1) $\phi(0), \phi(1), \ldots, \phi(x)$ satisfy the following linear equations:

$$
p_{0} q \phi(0)-p_{1} \phi(x-1)=\alpha,
$$

$$
\begin{aligned}
p_{0} q \phi & (u+1)+\left(q q_{0}+p p_{0} p(1)-1\right) \phi(u) \\
& +p \sum_{k=0}^{u-1} \phi(k) T(u-k)=\beta, \quad u=0,1,2, \ldots, x-1,
\end{aligned}
$$

where

$$
\begin{aligned}
\alpha=p q_{1} \sum_{k=0}^{x-1} \sum_{i=k+1}^{+\infty} f(k, i-k) T(i) \\
+p p_{1} \sum_{k=0}^{x-2} \sum_{i=k+1}^{+\infty} f(k, i-k) T(i)
\end{aligned}
$$




$$
\begin{array}{r}
+p \sum_{k=x}^{+\infty} \sum_{i=k+1}^{+\infty} f(k, i-k) J(i-1), \\
\beta=-p \sum_{k=u+1}^{\infty} f(k, k-u) J(k) ;
\end{array}
$$

(2) for $u \geq x$, the penalty functions $\phi(u)$ satisfy

$$
\begin{aligned}
\phi(u+1)= & \frac{1-q\left(q_{0} q_{1}+p_{0} p_{1}\right)}{q p_{0} q_{1}} \phi(u)-\frac{q_{0} p_{1}}{p_{0} q_{1}} \phi(u-1) \\
& -\frac{p}{q p_{0} q_{1}} \sum_{k=0}^{u} \phi(k) J(u-k-1) \\
& -\frac{p}{q p_{0} q_{1}} \sum_{k=u+1}^{+\infty} f(u, k-u) J(k-1), \\
\phi(u+1)= & \frac{q_{0} p_{1}}{p_{0} q_{1}} \phi(u)+\frac{p}{q p_{0} q_{1}} \sum_{k=0}^{u} \phi(k) \bar{J}(x-k-1) \\
& +\frac{p}{q p_{0} q_{1}} \sum_{k=u+1}^{+\infty} \sum_{i=k+1}^{+\infty} f(k, i-k) J(i-1) .
\end{aligned}
$$

Proof. Considering the insurance policy and dividend and claim number in the first time period $(0,1]$, there are twelve cases as follows:

(1) no insurance policy is obtained, no claim occurs, and no dividend is paid in $(0,1]$;

(2) an insurance policy is obtained, no claim occurs, and no dividend is paid in $(0,1]$;

(3) no insurance policy is obtained, a claim occurs, no dividend is paid, and $U(1) \geq 0$ in $(0,1]$;

(4) an insurance policy is obtained, a claim occurs, no dividend is paid, and $U(1) \geq 0$ in $(0,1]$;

(5) no insurance policy is obtained, a claim occurs, no dividend is paid, and $U(1)<0$ in $(0,1]$;

(6) an insurance policy is obtained, a claim occurs, no dividend is paid, and $U(1)<0$ in $(0,1]$;

(7) no insurance policy is obtained, no claim occurs, and a dividend is paid in $(0,1]$ (if $U(0)<x$, the case does not exist);

(8) an insurance policy is obtained, no claim occurs, and a dividend is paid in $(0,1]$ (if $U(0)<x$, the case does not exist);

(9) no insurance policy is obtained, a claim occurs, and a dividend is paid in $(0,1]$ (if $U(0)<x$, the case does not exist);

(10) an insurance policy is obtained, a claim occurs, a dividend is paid, and $U(1) \geq 0$ in $(0,1]$ (if $U(0)<x$, the case does not exist);
(11) no insurance policy is obtained, a claim occurs, a dividend is paid, and $U(1) \geq 0$ in $(0,1]$ (if $U(0)<x$, the case does not exist);

(12) an insurance policy is obtained, a claim occurs, a dividend is paid, and $U(1)<0$ in $(0,1]$ (if $U(0)<x$, the case does not exist).

Using the total probability formula, when $0 \leq u<x$,

$$
\begin{aligned}
\phi(u)= & q_{0} q \phi(u)+p_{0} q \phi(u+1)+\sum_{k=0}^{u} \phi(k) T(u-k) \\
& +p \sum_{k=u+1}^{+\infty} f(u, k-u) T(k) .
\end{aligned}
$$

Equation (13) is equivalent to (9).

When $u \geq x$,

$$
\begin{aligned}
\phi(u)=q[ & q_{0} p_{1} \phi(u-1)+\left(q_{0} q_{1}+p_{0} p_{1}\right) \phi(u) \\
& \left.+p_{0} q_{1} \phi(u+1)\right]+p \sum_{k=0}^{u} \phi(k) J(u-k-1) \\
& +p \sum_{k=u+1}^{+\infty} f(u, k-u) J(k-1) .
\end{aligned}
$$

Equation (11) comes from (14). Equation (14) is equivalent to

$$
\begin{aligned}
{\left[1-q\left(q_{0} q_{1}+p_{0} p_{1}\right)\right] \phi(u)=} & q q_{0} p_{1} \phi(u-1) \\
& +q p_{0} q_{1} \phi(u+1) \\
& +p \sum_{k=0}^{u} \phi(k) J(u-k-1) \\
& +p \sum_{k=u+1}^{+\infty} f(u, k-u) J(k-1) .
\end{aligned}
$$

Subtracting $q\left(q_{0} p_{1}+p_{0} q_{1}\right) \phi(u)$ from (15), we obtain

$$
\begin{aligned}
p \phi(u)= & q q_{0} p_{1}(\phi(u-1)-\phi(u)) \\
& +q p_{0} q_{1}(\phi(u+1)-\phi(u)) \\
& +p \sum_{k=0}^{u} \phi(k) J(u-k-1) \\
& +p \sum_{k=u+1}^{+\infty} f(u, k-u) J(k-1) .
\end{aligned}
$$

When $t \geq x$, summing (16) over $u$ from $x$ to $t$ yields

$$
\begin{aligned}
p \sum_{u=x}^{t} \phi(u)= & q q_{0} p_{1}(\phi(x-1)-\phi(t)) \\
& +q p_{0} q_{1}(\phi(t+1)-\phi(x))
\end{aligned}
$$




$$
\begin{aligned}
& +p \sum_{u=x}^{t} \sum_{k=0}^{u} \phi(k) J(u-k-1) \\
& +p \sum_{u=x}^{t} \sum_{k=u+1}^{+\infty} f(u, k-u) J(k-1) .
\end{aligned}
$$

Interchanging the summing order of the third term in the right-hand side of (17), we get

$$
\begin{aligned}
p \sum_{u=x}^{t} \sum_{k=0}^{u} \phi(k) J(u-k-1) & \\
= & p \sum_{k=0}^{t} \phi(k)(1-\bar{J}(t-k-1)) \\
& -p \sum_{k=0}^{x-1} \phi(k)(1-\bar{J}(x-k-2)) .
\end{aligned}
$$

Adding (18) to (17), we obtain

$$
\begin{aligned}
q q_{0} p_{1}( & \phi(x-1)-\phi(t))+q p_{0} q_{1}(\phi(t+1)-\phi(x)) \\
= & p \sum_{k=0}^{t} \phi(k) \bar{J}(t-k-1)-p \sum_{k=0}^{x-1} \phi(k) \bar{J}(x-k-2) \\
& -p \sum_{u=x}^{t} \sum_{k=u+1}^{+\infty} f(u, k-u) J(k-1) .
\end{aligned}
$$

The security loading $\delta>0$ leads to $\lim _{u \rightarrow+\infty} \psi(u)=0$. $f(x, y)$ is bounded function, $\phi(u) \leq \psi(u)\|f\|$, where $\|f\|=$ $\sup \{f(x, y) \mid x \in N, y \in N\}$. Therefore, $\lim _{u \rightarrow+\infty} \phi(u)=0$. By the Dominated Convergence Theorem, we can obtain

$$
\begin{aligned}
& p \sum_{k=0}^{t} \phi(k) \bar{J}(t-k-1) \\
& \quad \leq p \sum_{k=1}^{t+1} \phi(t+1-k) \bar{J}(k-2) \\
& \quad \leq p \sum_{k=1}^{\infty} \phi(t+1-k) \bar{J}(k-2) \longrightarrow 0 \quad(t \longrightarrow+\infty) .
\end{aligned}
$$

Let $t \rightarrow+\infty$ in (19), and we get

$$
\begin{aligned}
q q_{0} p_{1} \phi & (x-1)-q p_{0} q_{1} \phi(x) \\
= & -p \sum_{k=0}^{x-1} \phi(k) \bar{J}(x-k-2) \\
& -p \sum_{u=x}^{+\infty} \sum_{k=u+1}^{+\infty} f(u, k-u) J(k-1) .
\end{aligned}
$$

Subtracting (21) from (19)

$$
\begin{aligned}
& -q q_{0} p_{1} \phi(t)+q p_{0} q_{1} \phi(t+1) \\
& =p \sum_{k=0}^{t} \phi(k) \bar{J}(x-k-1) \\
& \quad+p \sum_{k=t+1}^{+\infty} \sum_{i=k+1}^{+\infty} f(k, i-k) J(i-1) .
\end{aligned}
$$

Equation (12) is equivalent to (22).

Subtracting $p_{0} q \phi(u+1)$ from both sides of (13), we obtain

$$
\begin{aligned}
p \phi(u)= & p_{0} q(\phi(u+1)-\phi(u))+p \sum_{k=0}^{u} \phi(k) T(u-k) \\
& +p \sum_{k=u+1}^{+\infty} f(u, k-u) T(k) .
\end{aligned}
$$

When $x \geq 1$, summing (23) over $u$ from 0 to $x-1$, we get

$$
\begin{aligned}
p \sum_{u=0}^{x-1} \phi(u)= & p_{0} q(\phi(x)-\phi(0))+p \sum_{u=0}^{x-1} \sum_{k=0}^{u} \phi(k) T(u-k) \\
& +p \sum_{u=0}^{x-1} \sum_{k=u+1}^{+\infty} f(u, k-u) T(k)
\end{aligned}
$$

Interchanging the summation order of the second term on the right-hand side of (24), (24) is equivalent to

$$
\begin{aligned}
p_{0} q(\phi(x)-\phi(0))= & p \sum_{k=0}^{x-1} \phi(k) \bar{J}(x-1-k) \\
& -p \sum_{u=0}^{x-1} \sum_{k=u+1}^{+\infty} f(u, k-u) T(k) .
\end{aligned}
$$

Replacing $x$ by $x-1$ and adding $p \phi(x-1)$ to both sides of (25), we yield

$$
\begin{aligned}
& p_{0} q(\phi(x)-\phi(0))+p \phi(x-1) \\
&= p \sum_{k=0}^{x-1} \phi(k) \bar{J}(x-1-k) \\
&-p \sum_{u=0}^{x-2} \sum_{k=u+1}^{+\infty} f(u, k-u) J(k) .
\end{aligned}
$$

From $q_{1} \times(25)+p_{1} \times(26)$, we obtain

$$
\begin{aligned}
q p_{0} q_{1} \phi(x)+\left(q p_{0} p_{1}+p p_{1}\right) \phi(x-1)-p_{0} q \phi(0) \\
=p \sum_{k=0}^{x-1} \phi(k) \bar{J}(t-k-2)-p q_{1} \sum_{k=0}^{x-1} \sum_{i=k+1}^{+\infty} f(k, i-k) J(i) \\
\quad-p p_{1} \sum_{k=0}^{x-2} \sum_{i=k+1}^{+\infty} f(k, i-k) J(i) .
\end{aligned}
$$

Equation (27) minus (21) is (9). The theorem has been proved. 
According to Theorem 1, $\phi(0), \phi(1), \ldots, \phi(x-1)$ can be obtained by solving the linear equations (8) and (9) when $x \geq 1$. And we can obtain $\phi(x+1), \phi(x+2), \ldots$ by (11). The following problem that needs to be solved is whether there is a unique solution to the set of linear equations (8) and (9).

Definition 2. Assume that the matrix $A=\left(a_{i j}\right) \in C^{n \times n}$, and it satisfies

$$
\left|a_{i i}\right|>\sum_{j \neq i}\left|a_{i j}\right| .
$$

Then $A$ is called a (row) strictly diagonally dominant matrix.
Lemma 3. If $A$ is a strictly diagonally dominant matrix, then $A$ is a nonsingular matrix.

Proof. For the proof, see [6].

Theorem 4. Under the assumption that the security loading $\delta>0$, the set of linear equations (8) and (9) have a unique solution.

Proof. Let $\phi=(\phi(0), \phi(1), \phi(2), \ldots, \phi(x)), \Delta=(\alpha, \beta(0)$, $\beta(1), \beta(2), \ldots, \beta(x)), \chi=q q_{0}+p p_{0} p(1)-1, \chi^{\prime}=p p_{0} p(1)-p$,

$$
B=\left(\begin{array}{cccccccc}
p_{0} q & 0 & 0 & 0 & \cdots & 0 & -p_{1} & 0 \\
\chi & p_{0} q & 0 & 0 & \cdots & 0 & 0 & 0 \\
p T(1) & \chi & p_{0} q & 0 & \cdots & 0 & 0 & 0 \\
p T(2) & p T(1) & \chi & p_{0} q & \cdots & 0 & 0 & 0 \\
\cdots & \cdots & \cdots & \cdots & \cdots & \cdots & \cdots & \cdots \\
p T(x-1) & p T(x-2) & p T(x-3) & p T(x-4) & \cdots & p T(1) & \chi & p_{0} q
\end{array}\right)
$$

then, the set of linear equations (8) and (9) can be rewritten as

$$
B \phi=\Delta .
$$

The coefficient matrix $B$ will be carried out by a series of elementary row operations as follows: the $(x+1)$ row is replaced by itself plus the first $x$ rows; the $x$ row is replaced by itself plus the first $x-1$ rows, and so on; the second row is replaced by itself plus the first row. The matrix $B$ is changed into

$$
\left(\begin{array}{ccccccccc}
p_{0} q & 0 & 0 & 0 & \cdots & 0 & 0 & -p_{1} & 0 \\
\chi^{\prime} & p_{0} q & 0 & 0 & \cdots & 0 & 0 & -p_{1} & 0 \\
p \bar{T}(1) & \chi^{\prime} & p_{0} q & 0 & \cdots & 0 & 0 & -p_{1} & 0 \\
p \bar{T}(2) & p \bar{T}(1) & \chi^{\prime} & p_{0} q & \cdots & 0 & 0 & -p_{1} & 0 \\
\cdots & \cdots & \cdots & \cdots & \cdots & \cdots & \cdots & \cdots & \cdots \\
p \bar{T}(x-2) & p \bar{T}(x-3) & p \bar{T}(x-4) & p \bar{T}(x-5) & \cdots & p \bar{T}(1) & \chi^{\prime} & p_{0} q-p_{1} & 0 \\
p \bar{T}(x-1) & p \bar{T}(x-2) & p \bar{T}(x-3) & p \bar{T}(x-4) & \cdots & p \bar{T}(2) & p \bar{T}(1) & \chi^{\prime}-p_{1} & p_{0} q
\end{array}\right) .
$$

For the first row, because $\delta>0$ and $\mu=\sum_{n=0}^{+\infty} \bar{P}(n)>1$,

$$
p_{0} q-p_{1}=p_{0}(1-p)-p_{1}>p_{0}-p_{1}-p \mu>0
$$

For the second row, because $q_{0} q+p p_{0} p(1)-1<0$, then

$$
\begin{aligned}
p_{0} q-\left|\chi^{\prime}\right|-p_{1} & =p_{0}-p p_{0} \bar{P}(1)-p-p_{1} \\
& >p_{0}-p(\bar{P}(1)+\bar{P}(0))-p_{1} \\
& >p_{0}-p \mu-p_{1}>0 .
\end{aligned}
$$

For the $i$ row $(2<i \leq x-1)$,

$$
p_{0} q-p \sum_{k=1}^{i-2} \bar{T}(k)+p p_{0} p(1)-p-p_{1}
$$

$$
\begin{aligned}
& =p_{0}-p p_{0} \sum_{k=1}^{i-1} \bar{P}(k)-p q_{0} \sum_{k=1}^{i-2} \bar{P}(k)-p-p_{0} \\
& \geq p_{0}-p p_{0}(\mu-1)-p q_{0}(\mu-1)-p-p_{0} \\
& =p_{0}-p \mu-p_{1}>0 .
\end{aligned}
$$


For the $x$ row, owing to $p_{0} q-p_{1}=p_{0}-p_{1}-p p_{0}>0$,

$$
\begin{aligned}
p_{0} q- & p_{1}-\left|\chi^{\prime}\right|-p \sum_{k=1}^{x-2} \bar{T}(k) \\
& =p_{0}-p_{0} p \sum_{k=1}^{x-2} \bar{P}(k)-q_{0} p \sum_{k=1}^{x-1} \bar{P}(k) \\
& \geq p_{0}-p p_{0}(\mu-1)-p q_{0}(\mu-1)-p-p_{0} \\
& =p_{0}-p \mu-p_{1}>0
\end{aligned}
$$

For $x-1$ row, owing to $p p_{0} p(1)-p-p_{1}<0$,

$$
\begin{aligned}
p_{0} q- & \left|\chi^{\prime}-p_{1}\right|-p \sum_{k=1}^{x-1} \bar{T}(k) \\
& =p_{0}-p-p_{1}-p p_{0} \sum_{k=1}^{x} \bar{P}(k)-p q_{0} \sum_{k=1}^{x-1} \bar{P}(k) \\
& \geq p_{0}-p p_{0}(\mu-1)-p q_{0}(\mu-1)-p_{1}-p \\
& =p_{0}-p \mu-p_{0}>0 .
\end{aligned}
$$

Thus, the matrix $B$ is a strictly diagonally dominant matrix. According to Lemma 3, matrix $B$ is a nonsingular matrix. So the set of linear equations have a unique solution. The theorem has been proved.

\section{The Asymptotic Estimate of the Penalty Function}

Let $D=\theta_{1} X_{1}+\epsilon_{1}-I_{1}$ denote the generating function of $D$; then

$$
G_{D}(r)=\left(p G_{X}+q\right)\left(p_{1} r+q_{1}\right)\left(\frac{p_{0}}{r}+q_{0}\right),
$$

where $G_{X}$ is the generating function of $X$.

Assumption 5. There exists a $r_{\infty}$ such that $G_{X}(r) \rightarrow+\infty$ $\left(r \rightarrow r_{\infty}\right)\left(r_{\infty}\right.$ is possibly $\left.+\infty\right)$.

This assumption is similar to the one in [11].

Let $G_{D}(r)=1$, and then

$$
\left(p G_{X}(r)+q\right)\left(p_{1} r+q_{1}\right)\left(p_{0}+q_{0} r\right)=r .
$$

Let $H(r)=\left(p G_{X}(r)+q\right)\left(p_{1} r+q_{1}\right)\left(p_{0}+q_{0} r\right) . H(0)=q q_{1} p_{0}$, $H(1)=1, H(r)$ is a convex and increasing function in $\left[0, r_{\infty}\right)$, and thus (38) has two real nonnegative roots at most, and one of them is 1 . Because $\delta>0, H^{\prime}(1)=1-\left(p_{0}-p \mu-p_{1}\right)<$ 1. Because $H^{\prime \prime}(r)>0$ in $\left[0, r_{\infty}\right), H(r)$ is strictly convex in $\left[0, r_{\infty}\right)$. Therefore, there exist two real roots in (38). Denote the other root by $R$, and then $R>1$.

Note that if $q_{0}=0$, (38) becomes

$$
\left(p G_{X}(r)+q\right)\left(p_{1} r+q_{1}\right)=r
$$

which is just the adjustment coefficient equation of the compound binomial model with randomized decisions on dividends payment (see [10]). The following lemma will be used to derive the asymptotic estimates of $\phi(u)$.
Lemma 6. $Z$ is a set of integers, $\left\{a_{k}, k \in Z\right\},\left\{b_{k}, k \in Z\right\}$, $\left\{u_{k}, k \in Z\right\}$ are sequences that satisfy $a_{k} \geq 0, \sum_{-\infty}^{+\infty} a_{k}=1$, $\sum_{-\infty}^{+\infty}|k| a_{k}<+\infty, \sum_{-\infty}^{+\infty} k a_{k}>0, \sum_{-\infty}^{+\infty}\left|b_{k}\right|<+\infty$, the greatest common divisor of the integers $k$ for which $a_{k}>0$ is 1 , and the bounded series $u_{k}$ satisfies the following renewal equation:

$$
u_{n}=\sum_{k=-\infty}^{+\infty} a_{n-k} u_{k}+b_{n}, \quad n=0, \pm 1, \pm 2, \ldots ;
$$

then $\lim _{n \rightarrow \infty} u_{n}$ and $\lim _{n \rightarrow-\infty} u_{n}$ exist. Furthermore, if $\lim _{n \rightarrow-\infty} u_{n}=0$, then

$$
\lim _{n \rightarrow \infty} u_{n}=\frac{\sum_{k=-\infty}^{\infty} b_{k}}{\sum_{k=-\infty}^{\infty} k a_{k}} .
$$

Proof. The proof can be seen in Karlin and Taylor [5] (Chapter 3).

Theorem 7. The asymptotic estimate for the penalty $\phi(u)$ is

$$
\phi(u) \sim K R^{-u},
$$

where

$$
\begin{gathered}
K=\left(\left[q p_{0} p_{1}(R-1)+p_{0} q R\left(R^{x}-1\right)\right] \phi(0)\right. \\
+(R-1)\left(q q_{0} p_{1}-q p_{0} p_{1}-p p_{1}\right) \sum_{m=1}^{x} R^{m} \phi(m-1) \\
\left.+K_{1}(R-1)\right) \times\left((R-1)\left(K_{2} R+p \sum_{k=2}^{+\infty} \bar{J}(k-2)\right)\right), \\
K_{2}=q q_{0} p_{1}+p p_{0} q_{1} \bar{P}(1)+p q_{0} p_{1}+p\left(p_{0} p_{1}+q_{0} q_{1}\right), \\
K_{3}=\sum_{m=1}^{x} R^{m} \sum_{k=0}^{m-1} \sum_{i=k+1}^{+\infty} f(k, i-k) T(i), \\
K_{4}=\sum_{m=1}^{x} R^{m} \sum_{k=0}^{m-2} \sum_{i=k+1}^{+\infty} f(k, i-k) T(i), \\
K_{5}=\sum_{m=x+1}^{+\infty} R^{m} \sum_{k=m+1}^{+\infty} f(k, i-k) J(i-1) .
\end{gathered}
$$

Proof. When $u>x$, from (12), we can obtain

$$
\begin{aligned}
\phi(u)= & \left(q_{0}+p_{0} p_{1}+p p_{1} q_{1}\right) \phi(u) \\
& +\left(q q_{0} p_{1}+p p_{0} q_{1} \bar{P}(1)+q_{0} p_{1} p+p\left(q_{0} q_{1}+p_{0} p_{1}\right)\right) \\
& \times \phi(u-1)+p \sum_{k=0}^{u-2} \phi(k) \bar{J}(u-2-k) \\
& +p \sum_{k=u}^{+\infty} \sum_{i=k+1}^{+\infty} f(k, i-k) J(i-1) .
\end{aligned}
$$


Equation (27) is equivalent to

$$
\begin{aligned}
\phi(u)= & \left(q_{0}+p_{0} p_{1}+p p_{1} q_{1}\right) \phi(u) \\
& +\left(q q_{0} p_{1}+p p_{0} q_{1} \bar{P}(1)+q_{0} p_{1} p+p\left(q_{0} q_{1}+p_{0} p_{1}\right)\right) \\
& \times \phi(u-1)+p \sum_{k=0}^{u-2} \phi(k) \bar{J}(u-2-k)+p_{0} q \phi(0) \\
& +\left(q q_{0} p_{1}-p_{0} p_{1} q-p p_{1}\right) \phi(u-1) \\
& -p q_{1} \sum_{k=0}^{u-1} \sum_{i=k+1}^{+\infty} f(k, i-k) T(i) \\
& -p p_{1} \sum_{k=0}^{u-2} \sum_{i=k+1}^{\infty} f(k, i-k) T(i) .
\end{aligned}
$$

Combining (44) and (45), we can obtain the renewal equation

$$
\begin{aligned}
\phi(u)= & \left(q_{0}+p_{0} p_{1}+p p_{1} q_{1}\right) \phi(u)+\left(q q_{0} p_{1}+p p_{0} q_{1}\right) \bar{P}(1) \\
& +q_{0} p_{1} p+p\left(q_{0} q_{1}+p_{0} p_{1}\right) \phi(u-1) \\
& +p \sum_{k=0}^{u-2} \phi(k) \bar{J}(u-2-k) \\
& +\left\{\begin{array}{l}
p_{0} q \phi(0)+\left(q q_{0} p_{1}-p_{0} p_{1} q-p p_{1}\right) \phi(u-1), \\
-p q_{1} \sum_{k=0}^{u-1} \sum_{i=k+1}^{+\infty} f(k, i-k) T(i) \\
-p p_{1} \sum_{k=0}^{u-2} \sum_{i=k+1}^{+\infty} f(k, i-k) T(i), \quad \\
p \sum_{k=u}^{+\infty} \sum_{i=k+1}^{+\infty} f(k, i-k) J(i-1), \quad x<u .
\end{array}\right.
\end{aligned}
$$

Denoting that $\bar{\phi}(u)=\phi(u) R^{u}$,

$$
a_{u}=\left\{\begin{array}{lr}
q_{0}+p_{0} p_{1}+p p_{1} q_{1}, & u=0, \\
R\left(q q_{0} p_{1}+p p_{0} q_{1} \bar{P}(1)+q_{0} p_{1} p+p\left(q_{0} q_{1}+p_{0} p_{1}\right)\right), & u=1, \\
p R^{u} \bar{J}(u-2), & u \geq 2,
\end{array}\right.
$$

$$
\begin{aligned}
& b_{u}=\left\{\begin{array}{lr}
q p_{0} q_{1}, & u=0, \\
p_{0} q \phi(0)+\left(q q_{0} p_{1}-p_{0} p_{1} q-p p_{1}\right) \phi(u-1) & \\
-p q_{1} \sum_{k=0}^{u-1} \sum_{i=k+1}^{+\infty} f(k, i-k) T(i) & 0<u \leq x, \\
-p p_{1} \sum_{k=0}^{u-2} \sum_{i=k+1}+\infty f(k, i-k) T(i), & x<u, \\
p \sum_{k=u}^{+\infty} \sum_{i=k+1}^{+\infty} f(k, i-k) J(i-1), &
\end{array}\right. \\
& b_{u}=p R^{u} \sum_{k=u}^{+\infty} \sum_{i=k+1}^{+\infty} f(k, i-k) J(i-1), \quad u>x .
\end{aligned}
$$

Multiplying (46) by $R^{u}$, we can obtain

$$
\bar{\phi}(u)=\sum_{k=0}^{u} a_{n-k} \bar{\phi}(k)+b_{u}, \quad u=0,1,2, \ldots
$$

We will prove that (48) satisfies the conditions of Lemma 6 . Consider

$$
\begin{aligned}
& \sum_{k=0}^{+\infty} a_{k}= q_{0}+p_{0} p_{1}+p p_{0} q_{1} \\
&+\left(q q_{0} p_{1}+p p_{0} q_{1} \bar{P}(1)+q_{0} p_{1} p+p\left(q_{0} q_{1}+p_{0} p_{1}\right)\right) R \\
&+\sum_{k=2}^{+\infty} p \bar{J}(k-2) R^{k} \\
&= q_{0}+p_{0} p_{1}+q_{0} p_{1} R+R^{2} p q_{0} p_{1} \sum_{k=0}^{+\infty} \bar{P}(k) R^{k} \\
&+p\left(q_{0} q_{1}+p_{0} p_{1}\right) \sum_{k=0}^{+\infty} \bar{P}(k) R^{k}+p p_{0} q_{1} \sum_{k=0}^{+\infty} \bar{P}(k) R^{k} \\
&= q_{0}+p_{0} p_{1}+q_{0} p_{1} R \\
&+\left(R^{2} p q_{0} p_{1}+p\left(q_{0} q_{1}+p_{0} p_{1}\right)+p p_{0} q_{1}\right) \frac{G_{X}(R)-1}{R-1} \\
&= 1, \quad 0 \leq b_{u} \geq p\|f\| R^{u} \sum_{k=u}^{+\infty} \bar{J}(k-1) ; \\
& \text { The following steps will prove that } \sum_{k=0}^{+\infty}\left|b_{k}\right|<\infty \text { is the root of }(38) . \\
& \text { where the last equation is valid because } x
\end{aligned}
$$


then

$$
\begin{aligned}
& \sum_{u=x+1}^{+\infty} b_{u} \leq p\|f\| \sum_{u=1}^{+\infty} R^{u} \sum_{k=u}^{+\infty} \bar{J}(k-1), \\
& p \sum_{u=1}^{+\infty} R^{u} \sum_{k=u}^{+\infty} \bar{J}(k-1)= p \sum_{k=1}^{+\infty} \sum_{u=1}^{k} \bar{J}(k-1) R^{u} \\
&= p \sum_{k=1}^{+\infty} \bar{J}(k-1) \frac{R^{k}-R}{R-1} \\
&= \frac{p}{R(R-1)} \sum_{k=2}^{\infty} R^{k} \bar{J}(k-2) \\
&-\frac{R}{R-1} \sum_{k=2}^{+\infty} \bar{J}(k-2) \\
& \leq \frac{p}{R(R-1)} \sum_{k=2}^{\infty} a_{k} \leq \frac{p}{R(R-1)}
\end{aligned}
$$

From (51), we can obtain $\sum_{u=x+1}^{+\infty} b_{u}<+\infty$. And because $\left|b_{k}\right|<$ $+\infty(0 \leq u \leq x), \sum_{u=0}^{+\infty} b_{u}<+\infty$. Further, we can get

$$
\begin{gathered}
\sum_{k=0}^{+\infty} b_{k}=\left[q p_{0} p_{1}+p_{0} q \frac{R\left(R^{x}-1\right)}{(R-1)} \phi(0)\right] \\
+\left(q q_{0} p_{1}-q p_{0} p_{1}-p p_{1}\right) \sum_{m=1}^{x} R^{m} \phi(m-1)+K_{1} \\
\sum_{k=1}^{+\infty} k a_{k}=K_{2}+p \sum_{k=2}^{+\infty} k R^{k} \bar{J}(k-2) .
\end{gathered}
$$

According to Lemma 6, we can derive

$$
\begin{aligned}
\lim _{u \rightarrow+\infty} \bar{\phi}= & \left(\left[q p_{0} p_{1}+p_{0} q \frac{R\left(R^{x}-1\right)}{R-1}\right] \phi(0)\right. \\
& \left.+\left(q q_{0} p_{1}-q p_{0} p_{1}-p p_{1}\right) \sum_{m=1}^{x} R^{m} \phi(m-1)+K_{1}\right) \\
& \times\left(K_{2} R+p \sum_{k=2}^{+\infty} k \bar{J}(k-2)\right)^{-1} .
\end{aligned}
$$

Equation (53) is equivalent to (42). The theorem has been proved.

\section{The Application of the Penalty Function}

We will give some examples of ruin quantities such as the ultimate ruin probability, the distribution of the surplus of the deficit at ruin to illustrate the application of the recursive formulas, and asymptotic estimates for the penalty function $\phi(u)$.
TABLE 1: Adjustment coefficients.

\begin{tabular}{ccccc}
\hline$P$ & $(0.9,0.015)$ & $(0.75,0.015)$ & $(0.75,0.055)$ & $(0.65,0.055)$ \\
$R$ & 1.02157 & 1.01547 & 1.01274 & 1.010447 \\
\hline
\end{tabular}

\subsection{Ruin Quantities}

Example 8. Let $f(x, y)=1, \phi(u)=\operatorname{Pr}(T<+\infty \mid U(0)=$ $u)=\psi(u)$, which is the ultimate ruin probability. By Theorem 1 , we can show that

(1) $\psi(0), \psi(1), \ldots, \psi(x)$ satisfy the following linear equations:

$$
\begin{gathered}
p_{0} q \psi(0)-p_{1} \psi(x-1)=\alpha, \\
p_{0} q \psi(u+1)+\left(q q_{0}+p p_{0} p(1)-1\right) \psi(u) \\
+p \sum_{k=0}^{u-1} \psi(k) T(u-k)=\beta, \\
u=0,1,2, \ldots, x-1,
\end{gathered}
$$

where

$$
\begin{gathered}
\alpha=p q_{1} \sum_{k=0}^{x-1} \bar{T}(k)+p p_{1} \sum_{k=0}^{x-2} \bar{T}(k)+p \sum_{k=x}^{+\infty} \bar{J}(u-1), \\
\beta=-p \bar{T}(u) ;
\end{gathered}
$$

(2) for $u \geq x$, the penalty functions $\phi(u)$ satisfy

$$
\begin{aligned}
\psi(u+1)= & \frac{1-q\left(q_{0} q_{1}+p_{0} p_{1}\right)}{q p_{0} q_{1}} \psi(u)-\frac{q_{0} p_{1}}{p_{0} q_{1}} \psi(u-1) \\
& -\frac{p}{q p_{0} q_{1}} \sum_{k=0}^{u} \psi(k) J(u-k-1)-\frac{p}{q p_{0} q_{1}} \bar{J}(k-1) .
\end{aligned}
$$

By Theorem 7, the asymptotic estimates of the ultimate ruin probability are

$$
\psi(u) \sim K_{\psi} R^{-u}
$$

where

$$
\begin{aligned}
K_{\psi}=( & {\left[q p_{0} p_{1}(R-1)+p_{0} q R\left(R^{x}-1\right)\right] \psi(0) } \\
& +(R-1)\left(q q_{0} p_{1}-q p_{0} p_{1}-p p_{1}\right) \\
& \left.\times \sum_{m=1}^{x} R^{m} \psi(m-1)+K_{1}(R-1)\right) \\
& \times\left((R-1)\left(K_{2} R+p \sum_{k=2}^{+\infty} \bar{J}(k-2)\right)\right)^{-1}
\end{aligned}
$$


TABLE 2: Exact values and asymptotic values for the ruin probability.

\begin{tabular}{|c|c|c|c|c|c|c|c|c|}
\hline \multirow{2}{*}{$u$} & \multicolumn{2}{|c|}{$P=(0.9,0.015)$} & \multicolumn{2}{|c|}{$P=(0.75,0.015)$} & \multicolumn{2}{|c|}{$P=(0.75,0.055)$} & \multicolumn{2}{|c|}{$P=(0.65,0.055)$} \\
\hline & E.V & A.V & E.V & A.V & E.V & A.V & E.V & A.V \\
\hline 0 & 0.5011 & - & 0.5684 & - & 0.5791 & - & 0.6804 & - \\
\hline 1 & 0.4992 & - & 0.5631 & - & 0.5747 & - & 0.6721 & - \\
\hline 2 & 0.4816 & - & 0.5589 & - & 0.5704 & - & 0.6665 & - \\
\hline 3 & 0.4781 & - & 0.5544 & - & 0.5658 & - & 0.6601 & - \\
\hline 4 & 0.4636 & - & 0.5392 & - & 0.5601 & - & 0.6565 & - \\
\hline 5 & 0.4601 & - & 0.5331 & - & 0.5548 & - & 0.6424 & - \\
\hline 6 & 0.4557 & 0.4701 & 0.5288 & 0.5324 & 0.5503 & 0.4831 & 0.6301 & 0.6417 \\
\hline 7 & 0.4516 & 0.4602 & 0.5034 & 0.5243 & 0.5482 & 0.5619 & 0.6295 & 0.6351 \\
\hline 8 & 0.4494 & 0.4505 & 0.4985 & 0.5163 & 0.5435 & 0.5549 & 0.6206 & 0.6285 \\
\hline 9 & 0.4373 & 0.4409 & 0.4916 & 0.5084 & 0.5401 & 0.5479 & 0.6156 & 0.6220 \\
\hline 10 & 0.4264 & 0.4316 & 0.4884 & 0.5007 & 0.5384 & 0.5410 & 0.6104 & 0.6156 \\
\hline 20 & 0.3095 & 0.3134 & 0.3856 & 0.3977 & 0.4452 & 0.4474 & 0.5243 & 0.5267 \\
\hline 30 & 0.2503 & 0.2532 & 0.3385 & 0.3411 & 0.3939 & 0.3942 & 0.4710 & 0.4747 \\
\hline 40 & 0.2027 & 0.2045 & 0.2914 & 0.2925 & 0.3465 & 0.3474 & 0.4254 & 0.4279 \\
\hline 50 & 0.1641 & 0.1652 & 0.2501 & 0.2509 & 0.3056 & 0.3060 & 0.3829 & 0.3856 \\
\hline 60 & 0.1332 & 0.1335 & 0.2152 & 0.2152 & 0.2695 & 0.2697 & 0.3467 & 0.3476 \\
\hline 80 & 0.0871 & 0.0871 & 0.1583 & 0.1583 & 0.2092 & 0.2093 & 0.2816 & 0.2823 \\
\hline 100 & 0.0568 & 0.0568 & 0.1164 & 0.1164 & 0.1625 & 0.1625 & 0.2292 & 0.2293 \\
\hline
\end{tabular}

$$
\begin{aligned}
& K_{1}=-p q_{1} K_{3}-p p_{1} K_{4}+K_{5}, \\
& K_{2}=q q_{0} p_{1}+p p_{0} q_{1} \bar{P}(1)+p q_{0} p_{1}+p\left(p_{0} p_{1}+q_{0} q_{1}\right), \\
& K_{3}=\sum_{m=1}^{x} R^{m} \sum_{k=0}^{m-1} \bar{T}(k), \quad K_{4}=\sum_{m=1}^{x} R^{m} \sum_{k=0}^{m-2} \bar{T}(k), \\
& K_{5}=\sum_{m=x+1}^{+\infty} R^{m} \bar{J}(m-1) .
\end{aligned}
$$

Example 9. Let $f(x, y)=I(y \leq z)(z=1,2, \ldots)$, and then $\phi(u)=\operatorname{Pr}((|U(T)| \leq z, T<+\infty \mid U(0)=u))=F(u, z)$, which is the distribution of the surplus of the deficit at ruin. By Theorem 1, we can show that

(1) $F(0, z), F(1, z), \ldots, F(x-1, z)$ satisfy the following linear equations:

$$
p_{0} q F(0, z)-p_{1} F(x-1, z)=\alpha,
$$

$p_{0} q F(u+1, z)+\left(q q_{0}+p p_{0} p(1)-1\right) F(u, z)$

$$
+p \sum_{k=0}^{u-1} \phi(k) T(u-k)=\beta, \quad u=0,1,2, \ldots, x-1,
$$

where

$$
\begin{gathered}
\alpha=p q_{1} \sum_{k=0}^{x-1} \bar{T}(k)-\bar{T}(k+z)+p p_{1} \sum_{k=0}^{x-2} \bar{T}(k) \\
-\bar{T}(k+z)+p \sum_{k=x}^{+\infty}(\bar{J}(k-1)-\bar{J}(k+z-1)) \\
\beta=-p(\bar{T}(u)-\bar{T}(u+z))
\end{gathered}
$$

(2) for $u \geq x$, the penalty functions $\phi(u)$ satisfy

$$
\begin{aligned}
F(u+1, z)= & \frac{1-q\left(q_{0} q_{1}+p_{0} p_{1}\right)}{q p_{0} q_{1}} F(u, z)-\frac{q_{0} p_{1}}{p_{0} q_{1}} F(u-1, z) \\
& -\frac{p}{q p_{0} q_{1}} \sum_{k=0}^{u} \phi(k) J(u-k-1) \\
& -\frac{p}{q p_{0} q_{1}}(\bar{J}(u-1)-\bar{J}(u+z-1)) .
\end{aligned}
$$

By Theorem 7, we can obtain the asymptotic estimates of the distribution function of deficit at ruin. Consider

$$
F(u, z) \sim K_{F}(z) R^{-u}
$$


TABLE 3: Exact values and asymptotic values for the distribution function of the deficit at ruin.

\begin{tabular}{|c|c|c|c|c|c|c|c|c|}
\hline \multirow{3}{*}{$u$} & \multicolumn{4}{|c|}{$P=(0.75,0.015)$} & \multicolumn{4}{|c|}{$P=(0.65,0.055)$} \\
\hline & \multicolumn{2}{|c|}{$z=10$} & \multicolumn{2}{|c|}{$z=15$} & \multicolumn{2}{|c|}{$z=10$} & \multicolumn{2}{|c|}{$z=15$} \\
\hline & E.V & A.V & E.V & A.V & E.V & A.V & E.V & A.V \\
\hline 0 & 0.6387 & - & 0.4492 & - & 0.6523 & - & 0.5457 & - \\
\hline 1 & 0.6236 & - & 0.4406 & - & 0.6434 & - & 0.5347 & - \\
\hline 2 & 0.6129 & - & 0.4351 & - & 0.6347 & - & 0.5238 & - \\
\hline 3 & 0.6012 & - & 0.4273 & - & 0.6265 & - & 0.5124 & - \\
\hline 4 & 0.5984 & - & 0.4198 & - & 0.6153 & - & 0.5004 & - \\
\hline 5 & 0.5837 & - & 0.4102 & - & 0.6048 & - & 0.4961 & - \\
\hline 6 & 0.5710 & 0.5894 & 0.4064 & 0.4219 & 0.5937 & 0.6174 & 0.4874 & 0.4984 \\
\hline 7 & 0.5654 & 0.5804 & 0.4009 & 0.4155 & 0.5895 & 0.6110 & 0.4806 & 0.4932 \\
\hline 8 & 0.5635 & 0.5716 & 0.3995 & 0.4091 & 0.5843 & 0.6047 & 0.4754 & 0.4881 \\
\hline 9 & 0.5578 & 0.5629 & 0.3953 & 0.4029 & 0.5804 & 0.5984 & 0.4705 & 0.4831 \\
\hline 10 & 0.5499 & 0.5543 & 0.3894 & 0.3968 & 0.5776 & 0.5923 & 0.4659 & 0.4781 \\
\hline 20 & 0.4359 & 0.4403 & 0.3103 & 0.3152 & 0.4971 & 0.5068 & 0.3947 & 0.4091 \\
\hline 30 & 0.3738 & 0.3776 & 0.2674 & 0.2703 & 0.4502 & 0.4567 & 0.3604 & 0.3687 \\
\hline 40 & 0.3201 & 0.3239 & 0.2286 & 0.2318 & 0.4074 & 0.4117 & 0.3294 & 0.3323 \\
\hline 50 & 0.2754 & 0.2778 & 0.1964 & 0.1988 & 0.3683 & 0.3710 & 0.2957 & 0.2995 \\
\hline 60 & 0.2364 & 0.2383 & 0.1688 & 0.1705 & 0.3305 & 0.3344 & 0.2673 & 0.2699 \\
\hline 80 & 0.1746 & 0.1753 & 0.1246 & 0.1255 & 0.2704 & 0.2716 & 0.2184 & 0.2193 \\
\hline 100 & 0.1287 & 0.1289 & 0.0920 & 0.0923 & 0.2206 & 0.2207 & 0.1779 & 0.1781 \\
\hline
\end{tabular}

where

$$
\begin{aligned}
& K_{F}(z)=( {\left[q p_{0} p_{1}(R-1)+p_{0} q R\left(R^{x}-1\right)\right] \phi(0) } \\
&+(R-1)\left(q q_{0} p_{1}-q p_{0} p_{1}-p p_{1}\right) \\
&\left.\times \sum_{m=1}^{x} R^{m} F(m-1, z)+K_{1}(R-1)\right) \\
&\left((R-1)\left(K_{2} R+p \sum_{k=2}^{+\infty} \bar{J}(k-2)\right)\right)^{-1} \\
& K_{2}=q q_{0} p_{1}+ p p_{0} q_{1} \bar{P}(1)+p q_{0} p_{1}+p\left(p_{0} p_{1}+q_{0} q_{1}\right), \\
& K_{3}= \sum_{m=1}^{x} R^{m} \sum_{k=0}^{m-1}(\bar{T}(k)-\bar{T}(k+z)) \\
& K_{4}= \sum_{m=1}^{x} R^{m} \sum_{k=0}^{m-2}(\bar{T}(k)-\bar{T}(k+z)) \\
& K_{5}=\sum_{m=}^{+\infty} x+1 \\
& R^{m}(\bar{J}(m-1)-\bar{J}(m+z-1)) .
\end{aligned}
$$

5.2. Numerical Illustration. The initial term $\phi(0), \phi(1), \ldots$, $\phi(x)$ can be obtained by solving the set of linear equations (8) and (9). $\phi(x+1), \phi(x+2), \ldots$ can be computed by two approaches, which are using the recursive formulas and asymptotic estimation, respectively. We will compare the asymptotic values for the ruin probability and distribution of the deficit at ruin with the exact values computed by the recursive formulas and analysis on the impact of the randomly paying dividends on the ruin probability and distribution of the deficit at ruin.

The numerical analysis will be performed using the following assumed parameters. The distribution of claim amount $X_{i}$ is a zero-truncated geometric distribution with parameter $\alpha=9 / 10$, and then $f(k)=(1-9 / 10)(9 / 10)^{i-1}$, $i=1,2, \ldots ; p=0.05$, and the threshold $x=5$. The four cases with the probability of paying dividend $P=\left(p_{0}, p_{1}\right)=$ $(0.9,0.015),(0.75,0.015),(0.75,0.055),(0.65,0.055)$ will be performed. The relative security loading are larger than zero in the four case, then there is a unique solution which is adjustment coefficient in each case. The adjustment coefficient $R$ is computed and shown in Table 1 .

The exact values calculated by recursive formulas (11)(12) and the asymptotic values estimated by (42) are shown in Table 2 and Table 3 for the ruin probability and the distribution of the deficit at ruin, respectively. In both of the tables, the E.V means the exact value, and the A.V means the asymptotic value.

From Table 3, we can find that the asymptotic values of ruin probability are generally close to the exact values with the surplus $u$ increasing under the cases $P=\left(p_{0}, p_{1}\right)=$ $(0.9,0.015),(0.75,0.015),(0.75,0.055),(0.65,0.055)$. It is easy to see that the ruin probability increases with decreasing probability of obtaining an insurance policy $p_{0}$, and increases with raising the probability of paying dividend $p_{1}$.

In Table 3, the exact values and asymptotic values for the distribution of the deficit at ruin when $z=10,15$ are shown. 
It suggests that the asymptotic values are more close to the exact values with the surplus increasing under the cases $P=$ $(0.75,0.015),(0.65,0.055)$.

\section{Conclusions}

In order to describe the surplus of the nonlife insurance companies reasonably, the compound binomial risk model with randomly charging premiums and paying dividends to shareholders is proposed in this paper. Further, we derive the recursive formulas and asymptotic estimation of penalty function using classical method. The results about penalty function are applied to obtain the recursive formulas and asymptotic estimations of the ruin probability and the distribution of the deficit at ruin. The numerical examples show that the actual penalty function can be approached by asymptotic estimation. The results about the model are meaningful to analyze the ruin problem about the joint-stock nonlife insurance companies. It may provide the reference for decision-making of the joint-stock nonlife insurance companies about risk management.

\section{Acknowledgments}

The authors would like to thank the editor and anonymous referees for their valuable comments and suggestions leading to improvement of this paper. This research is supported by the Natural Science Foundation of China under Grant no. 71203241.

\section{References}

[1] S. Cheng, H. U. Gerber, and E. S. W. Shiu, "Discounted probabilities and ruin theory in the compound binomial model," Insurance, vol. 26, no. 2-3, pp. 239-250, 2000.

[2] H. Cossette, D. Landriault, and E. Marceau, "Ruin probabilities in the compound Markov binomial model," Scandinavian Actuarial Journal, no. 4, pp. 301-323, 2003.

[3] D. C. M. Dickson, "Some comments on the compound binomial model," ASTIN Bulletin, vol. 24, no. 1, pp. 33-45, 1994.

[4] H. U. Gerber, "Mathematical fun with the compound Poisson process," ASTIN Bulletin, vol. 18, pp. 161-168, 1988.

[5] S. Karlin and H. M. Taylor, A First Course in Stochastic Processes, Academic Press, New York, NY, USA, 1975.

[6] N. J. Pullman, Matrix Theory and Its Applications, Marcel Dekker, New York, NY, USA, 1976.

[7] E. S. W. Shiu, "The probability of eventual ruin in compound binomial model," ASTIN Bulletin, vol. 19, pp. 179-190, 1989.

[8] Y. Xiao and J. Guo, "The compound binomial risk model with time-correlated claims," Insurance, vol. 41, no. 1, pp. 124-133, 2007.

[9] K. C. Yuen and J. Y. Guo, "Ruin probabilities for time-correlated claims in the compound binomial model," Insurance, vol. 29, no. 1, pp. 47-57, 2001.

[10] J. Tan and X. Yang, "The compound binomial model with randomized decisions on paying dividends," Insurance, vol. 39, no. 1, pp. 1-18, 2006.

[11] Z. Bao, "A note on the compound binomial model with randomized dividend strategy," Applied Mathematics and Computation, vol. 194, no. 1, pp. 276-286, 2007.
[12] D. Landriault, "Randomized dividends in the compound binomial model with a general premium rate," Scandinavian Actuarial Journal, no. 1, pp. 1-15, 2008.

[13] L. He and X. Yang, "The compound binomial model with randomly paying dividends to shareholders and policyholders," Insurance, vol. 46, no. 3, pp. 443-449, 2010.

[14] J. Tan and X. Yang, "The compound binomial model with a constant dividend barrier and periodically paid dividends," Journal of Systems Science \& Complexity, vol. 25, no. 1, pp. 167-177, 2012. 


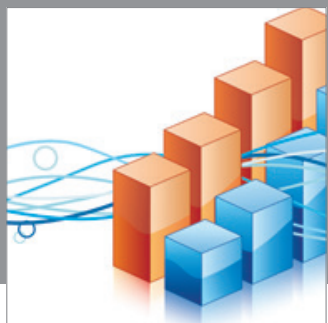

Advances in

Operations Research

mansans

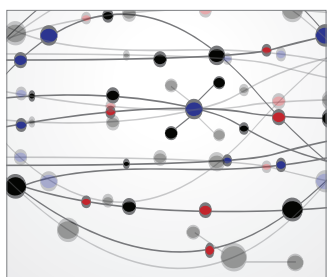

The Scientific World Journal
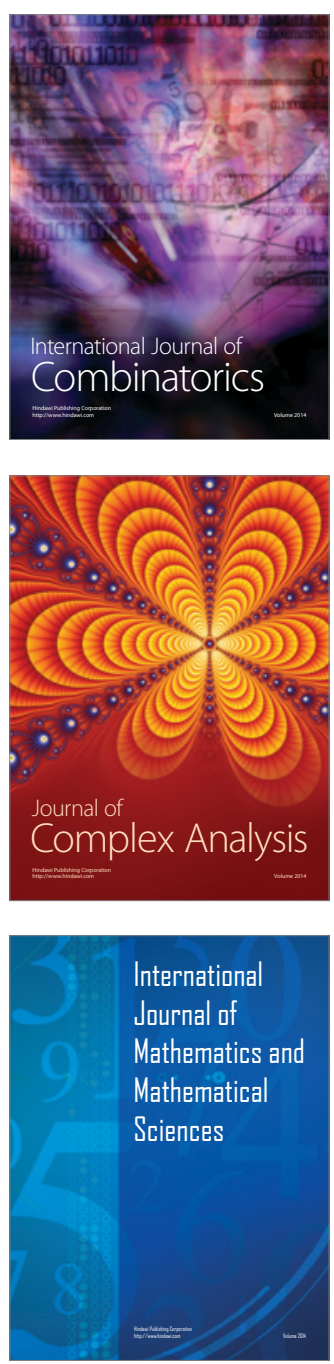
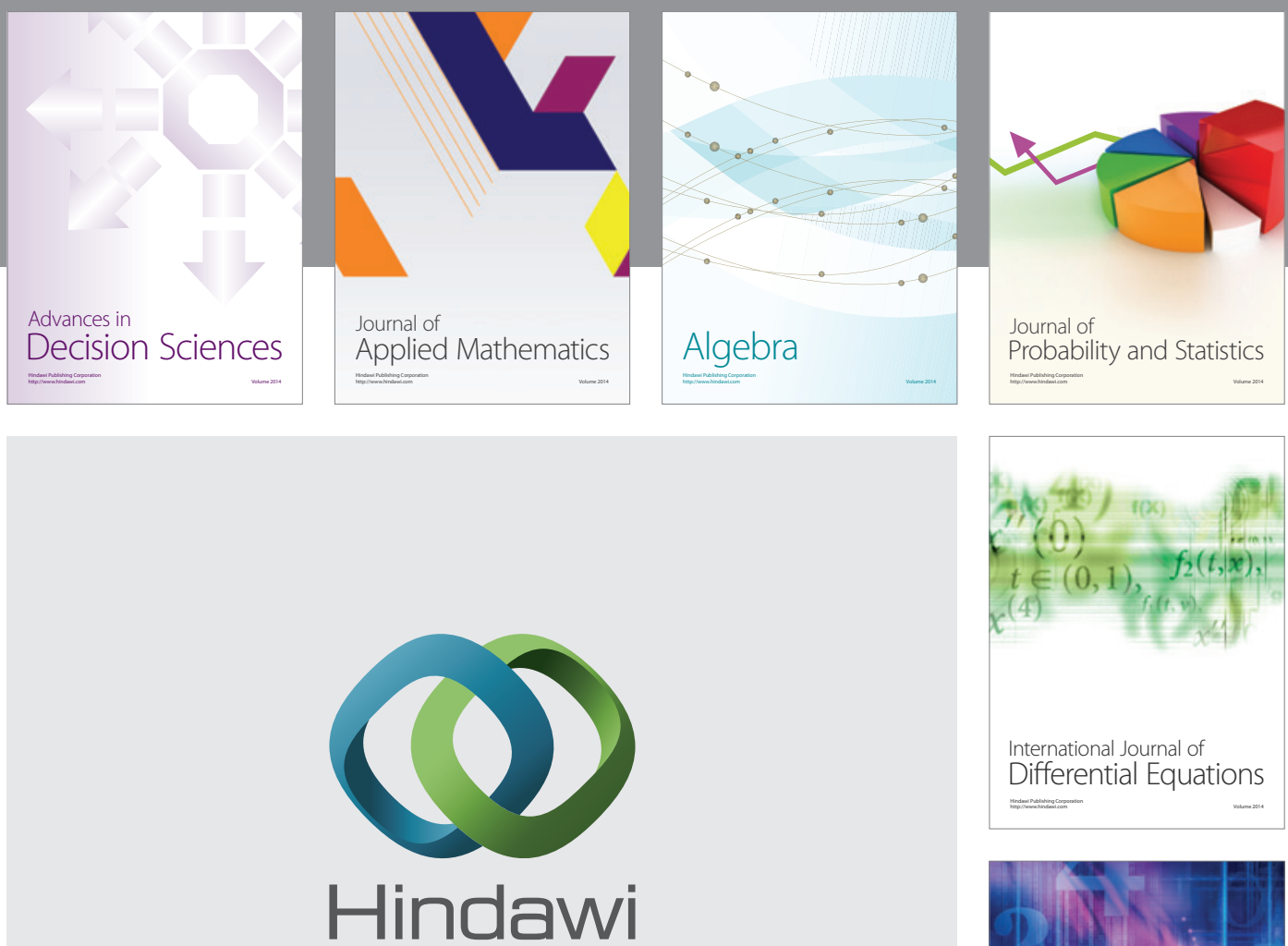

Submit your manuscripts at http://www.hindawi.com
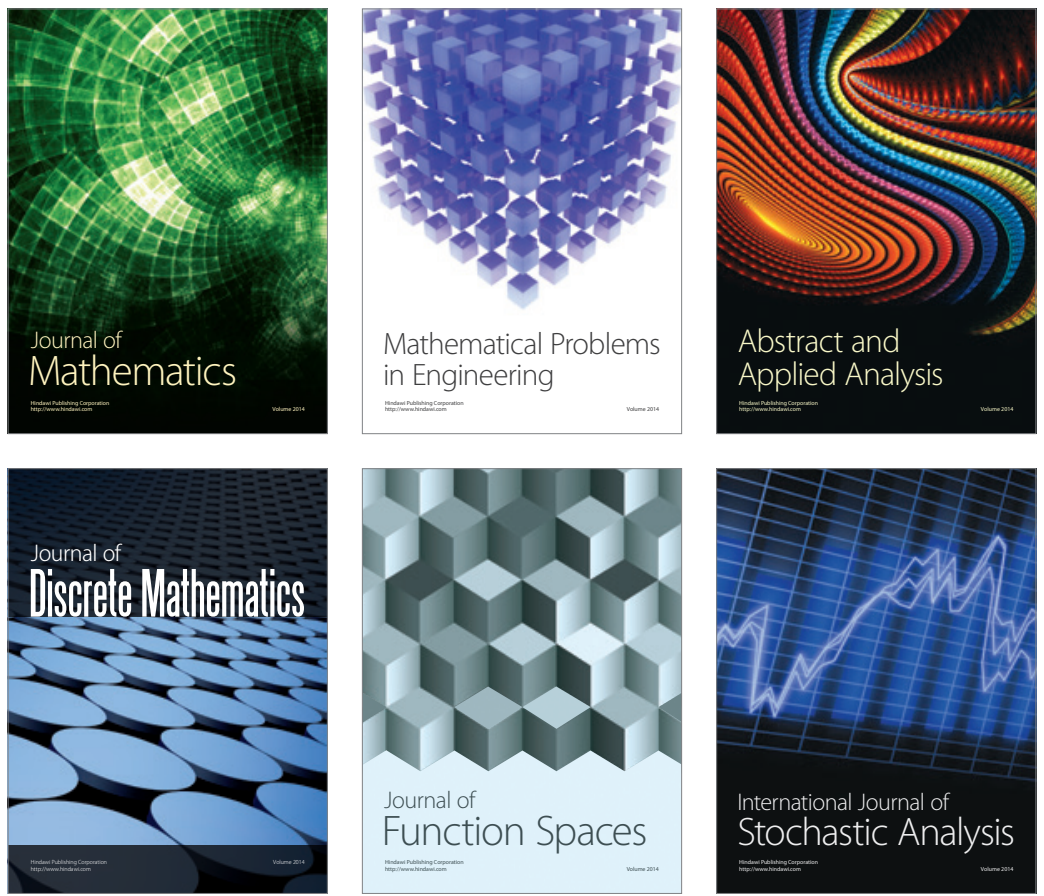

Journal of

Function Spaces

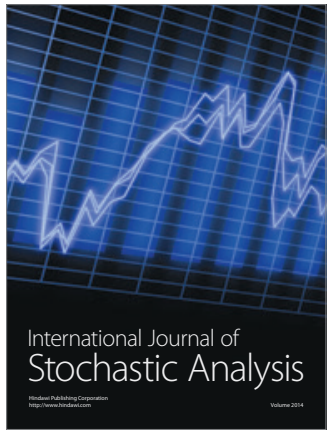

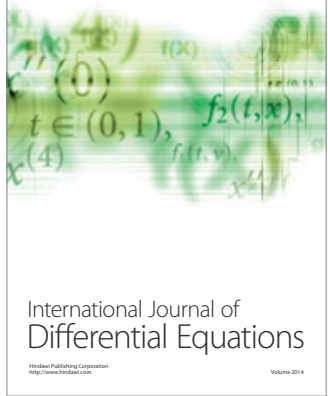
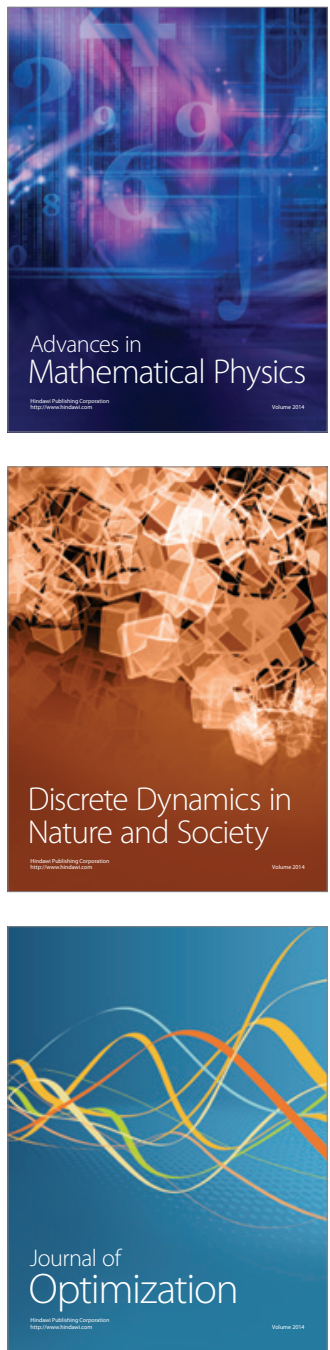